\title{
Simple procedure for phase-space measurement and entanglement validation
}

\author{
R. P. Rundle, ${ }^{1}$ P. W. Mills, ${ }^{1}$ Todd Tilma,${ }^{1,2}$ J. H. Samson, ${ }^{1}$ and M. J. Everitt ${ }^{1, *}$ \\ ${ }^{1}$ Quantum Systems Engineering Research Group \& Department of Physics, Loughborough University, \\ Leicestershire LE11 3TU, United Kingdom \\ ${ }^{2}$ Tokyo Institute of Technology, 2-12-1 Ookayama, Meguro-ku, Tokyo 152-8550, Japan \\ (Received 24 August 2016; revised manuscript received 28 April 2017; published 10 August 2017; \\ corrected 25 September 2017)
}

\begin{abstract}
It has recently been shown that it is possible to represent the complete quantum state of any system as a phase-space quasiprobability distribution (Wigner function) [Phys. Rev. Lett. 117, 180401 (2016)]. Such functions take the form of expectation values of an observable that has a direct analogy to displaced parity operators. In this work we give a procedure for the measurement of the Wigner function that should be applicable to any quantum system. We have applied our procedure to IBM's Quantum Experience five-qubit quantum processor to demonstrate that we can measure and generate the Wigner functions of two different Bell states as well as the five-qubit Greenberger-Horne-Zeilinger state. Because Wigner functions for spin systems are not unique, we define, compare, and contrast two distinct examples. We show how the use of these Wigner functions leads to an optimal method for quantum state analysis especially in the situation where specific characteristic features are of particular interest (such as for spin Schrödinger cat states). Furthermore we show that this analysis leads to straightforward, and potentially very efficient, entanglement test and state characterization methods.
\end{abstract}

DOI: 10.1103/PhysRevA.96.022117

\section{INTRODUCTION}

In 1932, Eugene Wigner, in an attempt to link the physics of many-particle systems (statistical physics) with quantum mechanics, defined a new way of describing the quantum state [1]. It took the form of a probability density function in position and momentum but, interestingly, it could take on negative values. Now named after its creator, the Wigner function is usually presented in advanced quantum optics texts as an integral combining the notions of Fourier transformations and autocorrelations. The function rapidly established its usefulness when its ability to take on negative values enabled physicists to be able to visualize quantum correlations in ways that were not previously possible. This capability is most commonly seen in the superposition of two macroscopically distinct coherent states [2-4]. In Fig. 1 we show an example of the Wigner function for such a superposition, the famous Schrödinger cat state. Such a state is very similar to those presented in Ref. [5] where it was demonstrated that nonclassical states of light can be made.

Similar schemes to those used in Ref. [5] for the direct reconstruction of the Wigner function for light have been in existence for some time (see, for example, Refs. [6-10]). These schemes all have the same feature that they, either implicitly or explicitly, rely on the fact that the Wigner function can be written as the expectation value of an appropriately normalized displaced parity operator or, equivalently, the expectation of parity for a displaced state [11]. In quantum mechanics, parity is similar to the usual notion of point reflection in that it maps a coordinate to one of opposite sign, the difference being that the coordinate in quantum mechanics is an observable operator. What this means is that, to reconstruct the Wigner function representation of the quantum state, all that is needed is a mechanism of displacing the quantum state and measuring its

\footnotetext{
*m.j.everitt@physics.org
}

parity. Such operations are well established in the quantum optics community [12]. A similar procedure, designed and built around finite-dimensional systems, is however still lacking.

To address this lack of a mechanism for displacing the quantum state and measuring its parity for finite-dimensional quantum systems, we propose a phase-space formalism that allows for a full representation of a product Hilbert space and offers easily understandable visualizations. Focusing on the latter, the symmetric-subspace approach, for example, the one presented in Ref. [13] where Wigner functions are constructed via a multipole expansion of spherical harmonics, is quite visually informative for harmonic-oscillator type systems $[14,15]$ and those with spin-1/2 symmetry. In more detail, it was Arecchi et al. [14] that first derived spin-1/2 atomic coherent states described by continuous functions of Euler angles. These states satisfied the same mathematical properties as the Glauber-Sudarshan infinite-dimensional coherent states $[2,16]$ but offered discreteness and Bloch symmetry to the corresponding Hilbert space, thus allowing them to be used to describe an assembly of spin- $j$ particles. Soon after, Agarwal [17] rewrote the Wigner $R$ and $P$ functions in terms of Arecchi's atomic coherent states, thus allowing for the study of various spin- $j$ systems under the Moyal quantization [18]. These parametrizations allow for easy visualizations of various quantum systems via Dicke state mappings [19,20] to a multipole expansion of spherical harmonics, for example, but they do not allow for a full representation of a product Hilbert space. As such, all symmetric-subspace Wigner functions are limited insofar that they cannot correctly show entanglement or represent the set of states that lie outside of this subspace (which, for large numbers, is nearly all of the Hilbert space).

To address the issue of the full representation of a product Hilbert space, we propose that the the phase space needs to be parametrized by more generalized coherent states such as those derived by Nemoto [21] and Mathur et al. [22]. Such states can be used to construct characteristic functions beyond those written with atomic or three-level coherent states [17,23-26]. 


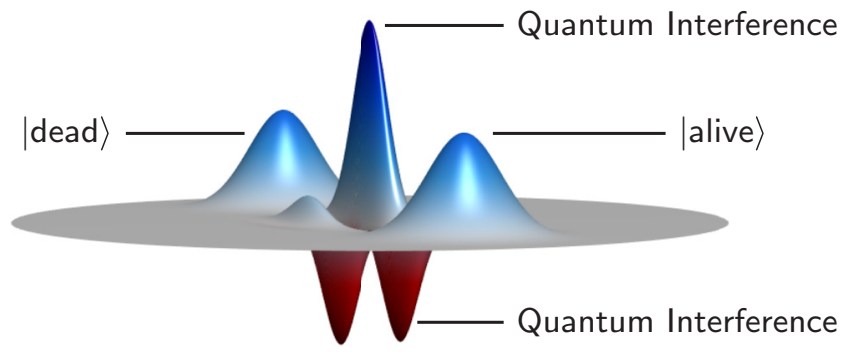

FIG. 1. The iconic textbook example of a Wigner function for a Schrödinger cat state. The bell shapes represent the "alive" and "dead" possible states for the "cat" and the oscillations between them indicate the quantum coherence between these states (i.e., the classic "both alive and dead" statement). A similar Wigner function without these interference terms would represent a state with a classical coin toss probability of being either alive or dead but not both. The presence of the interference terms indicates that this Wigner function represents a state that is in both states ("alive and dead") at the same time (a superposition).

These characteristic functions [27], by satisfying the Stratonovich-Weyl correspondence [28], are informationally complete $\mathrm{SU}(N)$-symmetric, spin- $j$ descriptions of finitedimensional quantum states ("qudits") [29-32]. This work is in contrast to that proposed by Wootters [33] and others for generating characteristic functions of $\mathrm{N}$-dimensional discrete systems. There, the motivating mathematics are built around analyzing "systems having only a finite number of orthogonal states. The 'phase space' for such a system is taken to be not continuous but discrete." [33]. The phase space generated by such generalized coherent states is continuous in its parametrization (see Refs. [34,35]), allows for Wigner functions to be generated by the methodology given in Ref. [36] (the expectation value of an appropriately normalized displaced general parity operator), can completely represent product Hilbert spaces of qudits (thus producing phase-space signatures of entanglement), and gives a method for visualizing said functions that is equivalent to that done for symmetric subspace representations, which we now discuss in more detail.

\section{BACKGROUND}

While it has been known for a long time that parity displacement could be done for continuous systems [16,37], following much work on the use of Wigner functions of discrete systems [17,18,23-27,30-33,38-44], it has only recently been proposed that any quantum system's Wigner function can be written as the expectation value of a displaced and/or rotated generalized parity operator [36]. Mathematically this can be expressed as

$$
\begin{aligned}
W_{\rho}(\boldsymbol{\Omega}) & =\left\langle U(\boldsymbol{\Omega}) \Pi U^{\dagger}(\boldsymbol{\Omega})\right\rangle_{\rho} \\
& =\operatorname{Tr}\left[\rho\left\{U(\boldsymbol{\Omega}) \Pi U^{\dagger}(\boldsymbol{\Omega})\right\}\right],
\end{aligned}
$$

where $W$ is the Wigner function and $\boldsymbol{\Omega}$ is the set of parameters over which displacement or rotations are defined (typically this would be position and momentum), $\rho$ is the density matrix, $U(\boldsymbol{\Omega})$ is a general displacement or rotation operator or collection of operators, and $\Pi$ 's definition is motivated by the usual parity operator. The conventional Wigner function in position and momentum space is obtained if $U$ is set to the displacement operator that defines coherent states $|\alpha\rangle$ from the vacuum state $|0\rangle$ according to $D(\alpha)|0\rangle=|\alpha\rangle$, and the operator $\Pi$ is defined to be twice the usual phase-space parity operator so that $\Pi|\alpha\rangle=2|-\alpha\rangle[45]$.

For a given system the choice of $U(\boldsymbol{\Omega})$ and $\Pi$ is not unique, but in Ref. [36] it was stipulated that a distribution $W_{\rho}(\Omega)$ over a phase space defined by the parameters $\Omega$ is a Wigner function of $\rho$ if there exists a kernel $\Delta(\Omega)$ [which we show can be written as a similarity transform with respect to a "displacement" of a parity-like operator, i.e., $\Delta(\Omega)=U(\boldsymbol{\Omega}) \Pi U^{\dagger}(\boldsymbol{\Omega})$ - and the Wigner function is the expectation value of this similarity-transformed operator] satisfying the following restricted version of the StratonovichWeyl correspondence (reproduced verbatim from Ref. [36]):

S-W.1 The mappings $\mathrm{W}_{\rho}(\boldsymbol{\Omega})=\operatorname{Tr}[\rho \Delta(\boldsymbol{\Omega})]$ and $\rho=$ $\int_{\boldsymbol{\Omega}} \mathrm{W}_{\rho}(\boldsymbol{\Omega}) \Delta(\boldsymbol{\Omega}) \mathrm{d} \boldsymbol{\Omega}$ exist and are informationally complete. Simply put, we can fully reconstruct $\rho$ from $\mathrm{W}_{\rho}(\boldsymbol{\Omega})$ and vice versa. ${ }^{1}$

$\mathrm{S}-\mathrm{W} .2 \mathrm{~W}_{\rho}(\boldsymbol{\Omega})$ is always real valued which means that $\Delta(\boldsymbol{\Omega})$ must be Hermitian.

S-W.3 $\mathrm{W}_{\rho}(\boldsymbol{\Omega})$ is "standardized" so that the definite integral over all space $\int_{\boldsymbol{\Omega}} \mathrm{W}_{\rho}(\boldsymbol{\Omega}) \mathrm{d} \boldsymbol{\Omega}=\operatorname{Tr} \rho$ exists and $\int_{\boldsymbol{\Omega}} \Delta(\boldsymbol{\Omega}) \mathrm{d} \boldsymbol{\Omega}=$ 11 .

S-W.4 Unique to Wigner functions, $\mathrm{W}_{\rho}(\boldsymbol{\Omega})$ is selfconjugate; the definite integral $\int_{\boldsymbol{\Omega}} \mathrm{W}_{\rho^{\prime}}(\boldsymbol{\Omega}) \mathrm{W}_{\rho^{\prime \prime}}(\boldsymbol{\Omega}) \mathrm{d} \boldsymbol{\Omega}=$ $\operatorname{Tr}\left[\rho^{\prime} \rho^{\prime \prime}\right]$ exists. This is a restriction of the usual StratonovichWeyl correspondence.

S-W.5 Covariance: Mathematically, any Wigner function generated by "rotated" operators $\Delta\left(\boldsymbol{\Omega}^{\prime}\right)$ (by some unitary transformation $V$ ) must be equivalent to rotated Wigner functions generated from the original operator $\left[\Delta\left(\boldsymbol{\Omega}^{\prime}\right) \equiv V \Delta(\boldsymbol{\Omega}) V^{\dagger}\right]$ i.e., if $\rho$ is invariant under global unitary operations then so is $\mathrm{W}_{\rho}(\boldsymbol{\Omega})$.

If we define $U(\boldsymbol{\Omega})$ as an element of a special unitary (SU) group that acts as a displacement or rotation and $\Pi$ as an appropriately normalized identity plus a traceless diagonal matrix (i.e., an element of the Cartan subalgebra of the appropriate group) then, from Ref. [36], Eq. (1) is sufficient to generate Wigner functions for any finite-dimensional, continuous-variable, quantum system. We note that, beyond satisfying the Stratonovich-Weyl correspondence, we have yet to fully determine the level to which this definition is constrained. Because $\Pi$ performs the same role as parity does in the standard Wigner function, we refer to it as an extended parity.

\section{THE SCHEME}

In this work we present a procedure for the measurement and reconstruction of the quantum state for a series of qubits from two different Wigner functions that both satisfy the above restricted Stratonovich-Weyl correspondence. We start by considering a Wigner function where the extended parity operator is defined with respect to the underlying group structure of the total system. We then proceed to investigate

\footnotetext{
${ }^{1}$ For the inverse condition, an intermediate linear transform may be necessary.
} 
another Wigner function, whose kernel comprises a tensor product of one-qubit kernels, which is arguably a more natural way of looking at composite quantum systems. In both cases we apply our procedure to IBM's Quantum Experience fivequbit quantum processor to demonstrate that we can measure and reconstruct the Wigner functions of two different Bell states and the five-qubit Greenberger-Horn-Zeilinger (GHZ) state.

While Wigner functions can be considered to be expectation values of displaced extended parity operators, this view does not necessarily lead to the best way to practically determine the Wigner function. As previously discussed, displacing the extended parity operator and taking its expectation value should be the same as displacing or rotating the state, i.e., creating a new "state":

$$
\tilde{\rho}(\boldsymbol{\Omega})=U^{\dagger}(\boldsymbol{\Omega}) \rho U(\boldsymbol{\Omega}),
$$

and calculating the expectation value of the unshifted extended parity operator

$$
\langle\Pi\rangle_{\tilde{\rho}(\boldsymbol{\Omega})}=\operatorname{Tr}[\tilde{\rho}(\boldsymbol{\Omega}) \Pi] .
$$

Mathematically this is equivalent to our original expression for the Wigner function [Eq. (1)] because trace is invariant under cyclic permutations of its arguments. Furthermore, it is possible, and in some cases (such as with the IBM Quantum Experience) easier, to make $\tilde{\rho}(\boldsymbol{\Omega})$ by performing local rotations on each qubit rather than displacing $\Pi$.

In the ideal case, the extended parity $\Pi$ shown in Eq. (3) will be directly measurable, allowing for reconstruction of the quantum state via its Wigner function without any intermediate steps being needed. Even if it is not possible to measure the extended parity directly, such as with the IBM Quantum Experience, there is a simple alternative. Note that $\Pi$, as introduced in Ref. [36], is always a diagonal operator in the computational basis. The Wigner function is then easy to calculate according to

$$
W(\boldsymbol{\Omega})=\sum_{n} \tilde{\rho}_{n n}(\boldsymbol{\Omega}) \Pi_{n n} .
$$

To determine the Wigner function we are only required to measure the probability of the rotated system occupying each state of the computational basis.

For a set of qubits the rotation of the system can be intuitively defined in terms of rotation operators acting on each of the system's constituent parts. Explicitly, we can define a total rotation operator for $N$ qubits as

$$
\mathbb{U}_{N}=\bigotimes_{i}^{N} U_{i}\left(\theta_{i}, \varphi_{i}, \Phi_{i}\right)
$$

where $U_{i}\left(\theta_{i}, \varphi_{i}, \Phi_{i}\right)=e^{\mathrm{i} \sigma_{z_{i}} \varphi_{i}} e^{\mathrm{i} \sigma_{y_{i}} \theta_{i}} e^{\mathrm{i} \sigma_{z_{i}} \Phi_{i}}$ is the $\mathrm{SU}(2)$ rotation operator for each qubit in terms of the Euler angles $\boldsymbol{\Omega}_{i}=$ $\left(\theta_{i}, \varphi_{i}, \Phi_{i}\right)$. In the following sections we discuss the Wigner functions defined through two different possible choices of $\Pi$.

\section{A SPIN WIGNER FUNCTION WITH SU(•) EXTENDED PARITY}

In this section we define and explore a Wigner function for $N$ qubits where the extended parity operator reflects the underlying group structure of the total system. Here, extended parity is motivated by the idea of doing what amounts to a global $\pi$ rotation on the hypersphere of the underlying $\mathrm{SU}\left(2^{[N]}\right)$ coherent-state representation. This is achieved by defining our extended parity operator $\Pi_{\mathrm{SU}\left(2^{[N]}\right)}$ as a $2^{N} \times 2^{N}$ diagonal matrix whose first element ${ }^{2}$ is $2^{-N}\left[1+\left(2^{N}-1\right) \sqrt{2^{N}+1}\right]$ and whose remaining diagonal elements are $2^{-N}\left[1-\sqrt{2^{N}+1}\right]$. For example,

$$
\Pi_{\mathrm{SU}\left(2^{[1]}\right)}=\frac{1}{2}\left(\begin{array}{cc}
1+\sqrt{3} & 0 \\
0 & 1-\sqrt{3}
\end{array}\right)=\frac{1}{2}\left[\mathbb{1}+\sqrt{3} \sigma_{z}\right]
$$

for one qubit and

$$
\begin{aligned}
\Pi_{\mathrm{SU}\left(2^{[2]}\right)} & =\frac{1}{4}\left(\begin{array}{cccc}
1+3 \sqrt{5} & 0 & 0 & 0 \\
0 & 1-\sqrt{5} & 0 & 0 \\
0 & 0 & 1-\sqrt{5} & 0 \\
0 & 0 & 0 & 1-\sqrt{5}
\end{array}\right) \\
& =\frac{1}{4}\left[\mathbb{1} \otimes \mathbb{1}+\sqrt{5} \mathbb{1} \otimes \sigma_{z}+\sqrt{5} \sigma_{z} \otimes \mathbb{1}+\sqrt{5} \sigma_{z} \otimes \sigma_{z}\right]
\end{aligned}
$$

for two qubits in the computational basis.

Combining this definition of extended parity with the composite rotation operator $\mathbb{U}_{N}$, we obtain the kernel

$$
\Delta_{\mathrm{SU}\left(2^{[N]}\right)}\left(\left\{\theta_{i}, \varphi_{i}\right\}\right)=\mathbb{U}_{N} \hat{\Pi}_{\mathrm{SU}\left(2^{[N]}\right)} \mathbb{U}_{N}^{\dagger}
$$

that satisfies the restricted Stratonovich-Weyl correspondence given in the introduction. We note that the $\Phi_{i}$ make no contribution because $\Pi_{\mathrm{SU}\left(2^{[N]}\right)}$ commutes with $\sigma_{z_{i}}$. This kernel defines our $\mathrm{SU}\left(2^{[N]}\right)$ extended parity-based Wigner function according to

$$
W_{\mathrm{SU}\left(2^{[N]}\right)}\left(\left\{\theta_{i}, \varphi_{i}\right\}\right)=\operatorname{Tr}\left[\rho \mathbb{U}_{N} \hat{\Pi}_{\mathrm{SU}\left(2^{[N]}\right)} \mathbb{U}_{N}^{\dagger}\right] .
$$

Let us now consider the specific case of the Wigner function $W_{\mathrm{SU}\left(2^{[N]}\right)}$ for two qubits. Each qubit brings with it two degrees of freedom expressed in terms of Euler angles $\boldsymbol{\Omega}=\left(\theta_{1}, \varphi_{1}, \theta_{2}, \varphi_{2}\right)$; thus the associated Wigner function takes the form of a four-dimensional quasiprobability distribution $W_{\mathrm{SU}\left(2^{[2]}\right)}\left(\theta_{1}, \varphi_{1}, \theta_{2}, \varphi_{2}\right)$. Four-dimensional functions are not easy to visualize, but we can take slices of the function in order to gain an appreciation of it as a whole. In Figs. 2(a)-2(d) we show some example Wigner function slices for two Bell states. Specifically, Figs. 2(a) and 2(b) show the equal-angle ("= "४) slice $W_{\mathrm{SU}\left(2^{[2]}\right)}^{=\varangle}(\theta, \varphi)=W_{\mathrm{SU}\left(2^{[2]}\right)}(\theta, \varphi, \theta, \varphi)$ while Figs. 2(c) and 2(d) show the slice $W_{\mathrm{SU}\left(2^{[2]}\right)}^{\varphi_{i}=0}\left(\theta_{1}, \theta_{2}\right)=$ $W_{\mathrm{SU}\left(2^{[2]}\right)}\left(\theta_{1}, 0, \theta_{2}, 0\right)$. Note that Figs. 2(e) and 2(f) will be discussed in Sec. V, while more general two-qubit Wigner function dynamics are shown in Ref. [46].

\footnotetext{
${ }^{2}$ This particular representation of extended parity is a rotation of the extended parity operator given in Ref. [36] that we have taken in order to keep within the conventions of the experimental physics and quantum information communities. As with the extended parity operator given in Ref. [36], ours is still a linear function of the identity plus the Cartan subalgebra of the selfsame SU group.
} 


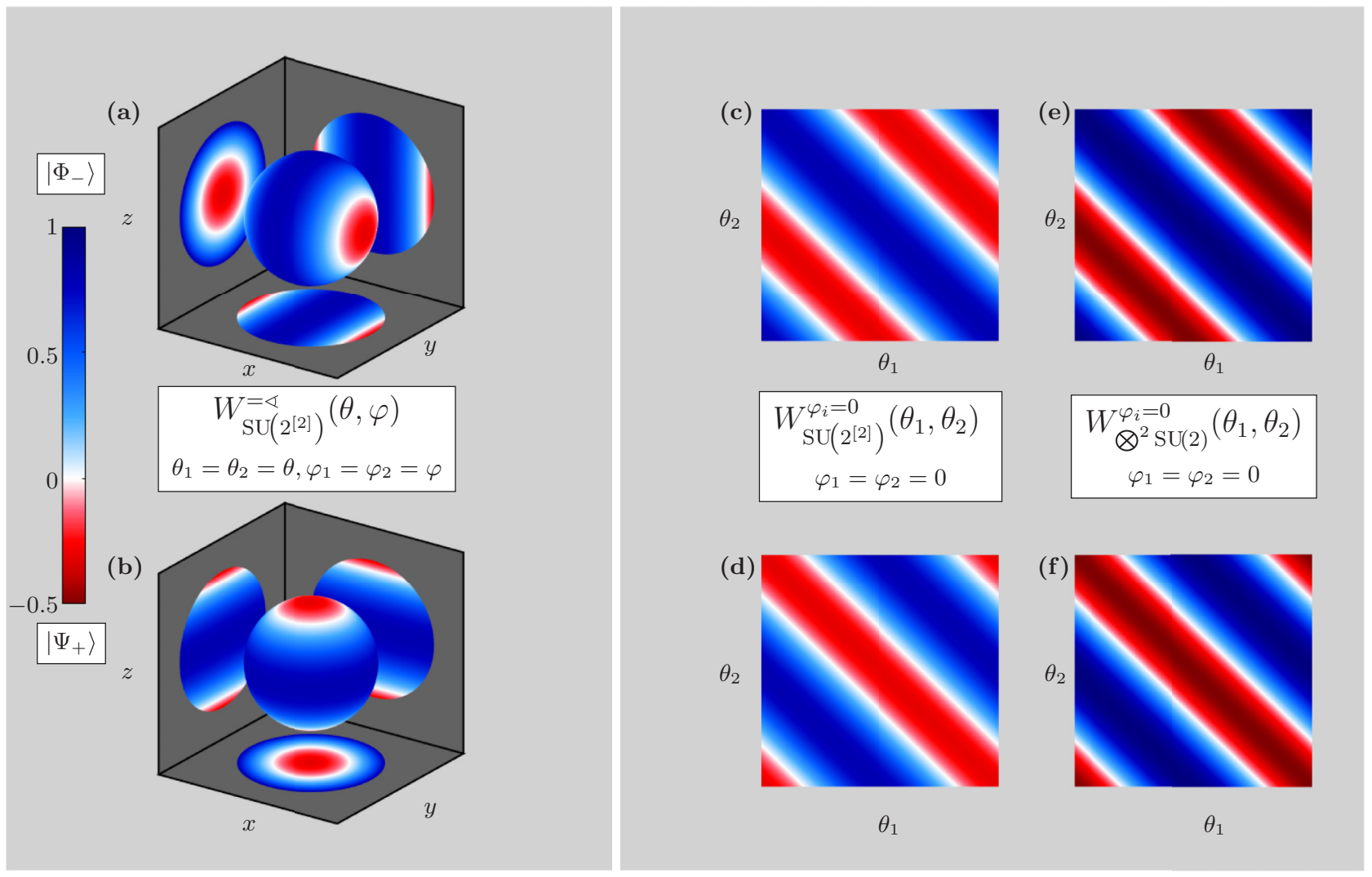

FIG. 2. (a)-(d) Slices from the four-dimensional Wigner function $W_{\mathrm{SU}\left(2^{[2]}\right)}\left(\theta_{1}, \varphi_{1}, \theta_{2}, \varphi_{2}\right)$ of two qubits for two different, maximally entangled, Bell states $\left|\Phi_{-}\right\rangle=\left(|0\rangle_{1}|0\rangle_{2}-|1\rangle_{1}|1\rangle_{2}\right) / \sqrt{2}$ and $\left|\Psi_{+}\right\rangle=\left(|0\rangle_{1}|1\rangle_{2}+|1\rangle_{1}|0\rangle_{2}\right) / \sqrt{2}$. The three-dimensional plots in panels (a) and (b) show $W_{\mathrm{SU}\left({ }^{[2]}\right)}^{=\varangle}(\theta, \varphi)$, the slice where $\theta=\theta_{1}=\theta_{2}$ and $\varphi=\varphi_{1}=\varphi_{2}$. The two-dimensional plots in panels (c) and (d) of $\theta_{1}$ versus $\theta_{2}$ show $W_{\mathrm{SU}\left(2^{[2]}\right)}^{\varphi_{i}=0}\left(\theta_{1}, \theta_{2}\right)$, the slice where $\varphi_{1}=\varphi_{2}=0$. We recommend that the reader see the Supplemental Material [46], which expands on these figures and shows animations of the Deutsch-Jozsa algorithm [47] and the creation of all four Bell states (in the animations, for example, it becomes clear that the Wigner functions for the Bell states, or, for that matter, any maximally entangled two-qubit state, are simply rotations of the same function in four-dimensional space). Later in this work we present experimental reconstructions of the $\theta_{1}$ versus $\theta_{2}$ plots. In understanding the form of these plots we note that the $\left|\Psi_{+}\right\rangle$state is one with total spin-angular momentum $\hbar$ but zero total $z$ spin-angular momentum. We thus expect to see the observed ring-like symmetry in $W_{\mathrm{SU}\left(2^{[N]}\right)}^{=\varangle,}(\theta)$ for $\left|\Psi_{+}\right\rangle$(the symmetry of $\left|\Phi_{-}\right\rangle$ follows from $\left|\Psi_{+}\right\rangle$because they are rotations of each other in four-dimensional space). This state is also an angular-momentum analog of a photon-number (Fock) state which shares a similar symmetry in its Wigner function [8-10]. In panels (e) and (f) we show $W_{\otimes^{2}}^{\varphi_{i}=0}\left(\theta_{1}, \theta_{2}\right)$ created by using the alternative extended parity operator $\Pi_{\otimes^{2} \mathrm{SU}(2)}$ as discussed in Sec. V. The availability of more than one extended parity operator, which produces Wigner functions with qualitatively very similar features, opens up possible alternative paths for direct phase-space reconstruction (note we have also included an animation of $W_{\otimes^{2} \text { SU(2) }}$ for the creation of the Bell states in the Supplemental Material [46]).

To demonstrate that this function is indeed easy to construct we have taken advantage of IBM's Quantum Experience project. The project makes available through the Internet a five-qubit processor, initially based on a simple "star" topology: ${ }^{3}$ a central qubit is coupled to four other qubits. The machine has already been used to produce interesting results $[48,49]$. Here we use it to measure and reconstruct the Wigner functions for the two Bell states $\left|\Phi_{+}\right\rangle$and $\left|\Psi_{-}\right\rangle$, as presented in Fig. 2. In this work, we are limited by the operations that IBM has made available to the user, operations that naturally focus on quantum computing applications. Nevertheless, following Eq. (2), we are able to produce

${ }^{3}$ Before the early-2017 update by IBM. $\tilde{\rho}\left(\theta_{1}, \varphi_{1}, \theta_{2}, \varphi_{2}\right)$ by using rotations generated by combinations of gate operations and readout state populations of $\tilde{\rho}_{n n}\left(\theta_{1}, \varphi_{1}, \theta_{2}, \varphi_{2}\right)$ via the standard output of the IBM processor. We then use Eqs. (4) and (7) to reconstruct the Wigner function (9).

In Fig. 3 we plot the Wigner function $W_{\mathrm{SU}\left(2^{[2]}\right)}^{\varphi_{i}=0}\left(\theta_{1}, \theta_{2}\right)$ slices, comparing the ideal theoretical values of Figs. 2(c) and 2(d), values generated by IBM's built in simulator (that models environmental effects) and real experimental data. The calibration data pertaining to the experiments are provided in Table I. In principle, to fully reconstruct the state requires us to measure the same number of points as needed to reconstruct the density matrix. In Fig. 3 we have actually measured more, and different, points than would be needed to fully reconstruct the state. This was done to demonstrate the ability to generate 


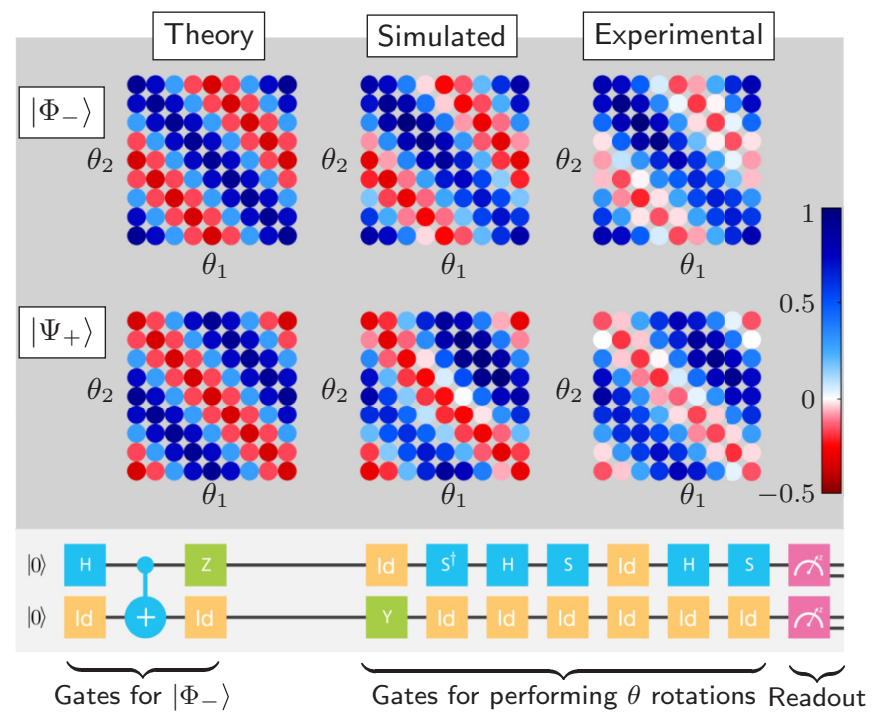

FIG. 3. Plots of the spin Wigner function for the two Bell states $\left|\Phi_{+}\right\rangle$and $\left|\Psi_{-}\right\rangle$. We plot $\theta_{1}$ versus $\theta_{2}$ for the $W_{\mathrm{SU}\left(2^{[2]}\right)}^{\varphi_{i}=0}$ slice of the Wigner function for two qubits; making use of the periodicity of the function at the edges of each plot for computational efficiency. We have included for comparison ideal theoretical values, numerical results using IBM's built in simulator, and real experimental data from IBM's quantum processor. The quantum circuit presented above is a screenshot taken directly from IBM's Quantum Experience web interface. It provides an example of the measurement protocol we used to obtain the diagonal elements of the rotated density matrix $\tilde{\rho}_{n n}\left(\theta_{1}, \varphi_{1}, \theta_{2}, \varphi_{2}\right)$. The theoretical, simulated, and experimental data are all in very good agreement with each other. Slight differences exist due to imperfect implementation of needed rotations due to different gate operations having different levels of noise (decoherence). It should be straightforward to replace the "Gates for performing $\theta$ rotations" with generalized rotation operators on each qubit. Furthermore, if measurement of the extended parity operator ( $\Pi)$ were available, direct observation of the quantum state would be reduced to a two-stage process of rotate and measure. We believe that such a protocol, because it would need fewer gate operations, would result in better agreement between theory and experiment than that seen in this figure. Note that, in order to have good color graduation in the transition from positive to negative values, there is some color clipping for the very strong blue points.

the Wigner function by using a raster scan approach because this makes clear the straightforward nature of our measurement method. Due to finite computational resources, and the need to do rotations as outlined above, we are limited in our resolution. Nevertheless, we find good agreement between theory, simulation, and experimental data, demonstrating that our tomographic process is clearly able to distinguish between the two Bell states.

Bell states are interesting both as an example of maximally entangled states and for their usefulness in quantum information processing. Fortunately, for systems comprising more spins, we can extend this class of states to those that have a direct analogy with optical Schrödinger cat states as considered in Ref. [5] and others. Such states are termed "spin-cat states" of which the GHZ state [50] is an excellent example. In previous theoretical work, spin
TABLE I. Calibration data for the experimental results contained within this paper. Data for the Bell state and GHZ Wigner functions were taken on June 16 and 17, 2016 when the fridge temperature was 18.25 and $17.916 \mathrm{mK}$, respectively. $T_{1}$ and $T_{2}$ are the usual relaxation times, $\epsilon_{g}$ is the gate error, $\epsilon_{r}$ is the readout error and $\epsilon_{g}^{i 2}$ is the CNOT gate error between the qubit listed and qubit 2 (which is the target qubit for the CNOT operation).

\begin{tabular}{|c|c|c|c|c|c|c|c|}
\hline \multirow[b]{2}{*}{ Qubit } & \multicolumn{2}{|c|}{ Bell } & \multicolumn{5}{|c|}{ GHZ } \\
\hline & 1 & 2 & 0 & 1 & 2 & 3 & 3 \\
\hline$T_{1}(\mu \mathrm{s})$ & 85.8 & 75.1 & 58.9 & 87.1 & 74.7 & 74.8 & 65.5 \\
\hline$T_{2}(\mu \mathrm{s})$ & 109.6 & 58.8 & 74.8 & 142.2 & 59.2 & 53.2 & 48.4 \\
\hline$\epsilon_{g}\left(\times 10^{-2}\right)$ & 0.15 & 0.2 & 0.29 & 0.2 & 0.23 & 0.23 & 0.89 \\
\hline$\epsilon_{r}\left(\times 10^{-2}\right)$ & 4.6 & 4.3 & 4.6 & 4.2 & 3.6 & 3.6 & 5.7 \\
\hline$\epsilon_{g}^{i 2}\left(\times 10^{-2}\right)$ & 3.19 & & 5.21 & 3.31 & & 3.18 & 6.55 \\
\hline
\end{tabular}

Wigner-like functions have been proposed as a mechanism for visualizing such cat states [13,51,52]. In analogy with measuring Wigner functions of nonclassical cavity field states [5], using our method we now construct the $W_{\mathrm{SU}\left(2^{[5]}\right)}$ Wigner function for a spin-cat of the form $\left|\mathrm{GHZ}_{5}\right\rangle=$ $\left(|0\rangle_{1}|0\rangle_{2}|0\rangle_{3}|0\rangle_{4}|0\rangle_{5}-|1\rangle_{1}|1\rangle_{2}|1\rangle_{3}|1\rangle_{4}|1\rangle_{5}\right) / \sqrt{2}$. In Fig. 4 we show the $\theta_{1}=\theta_{2}=\theta_{3}=\theta_{4}=\theta_{5}$ and $\varphi_{1}=\varphi_{2}=\varphi_{3}=\varphi_{4}=$ $\varphi_{5}$ slice of the $W_{\mathrm{SU}\left(2^{[5]}\right)}$ Wigner function for $\left|\mathrm{GHZ}_{5}\right\rangle$ which is the higher-dimensional analog of Figs. 2(a) and 2(b). We show both theoretical predictions and, due to limited computational resources, as insets, simulation and experimental data obtained from the IBM machine. Once more the calibration data pertaining to the experiments is provided in Table I. We note that the $\theta_{1}=\theta_{2}=\theta_{3}=\theta_{4}=\theta_{5}$ and $\varphi_{1}=\varphi_{2}=\varphi_{3}=$ $\varphi_{4}=\varphi_{5}$ slice does not contain all the information needed to reconstruct the state; for full reconstruction we would need to measure and visualize all $\left\{\theta_{i}, \theta_{j}\right\} i \neq j$ sets of angles for various values of $\varphi_{i}$. For the top and bottom point the theoretical value is 2.7 while the simulated values are 1.64 and 1.70, and experimental values 1.16 and 1.22 , respectively. Here simulation and experiment are in good agreement. The difference from the theoretical values for all four points indicates that there is some decoherence and/or gate and measurement errors in the system, mostly accounted for in IBM's simulation, meaning that the observed state is not in an ideal GHZ state.

\section{A WIGNER FUNCTION FOR TENSOR PRODUCTS OF SPINS}

The Stratonovich-Weyl conditions do not uniquely specify the extended parity operator $\Pi$ and hence the Wigner function is also not uniquely defined. Because of this, it is natural to ask what difference choosing alternative Wigner functions will make. As our current focus is on experimental reconstruction of the quantum state in phase space, we believe that it is instructive to explore at least one alternative whose direct measurement may be more readily available to those working in quantum information. In the previous case, the definition of extended parity was motivated by the idea of a global $\pi$ rotation on the hypersphere of the underlying $\mathrm{SU}\left(2^{[N]}\right)$ coherent-state representation. In this case the notion of extended parity is 


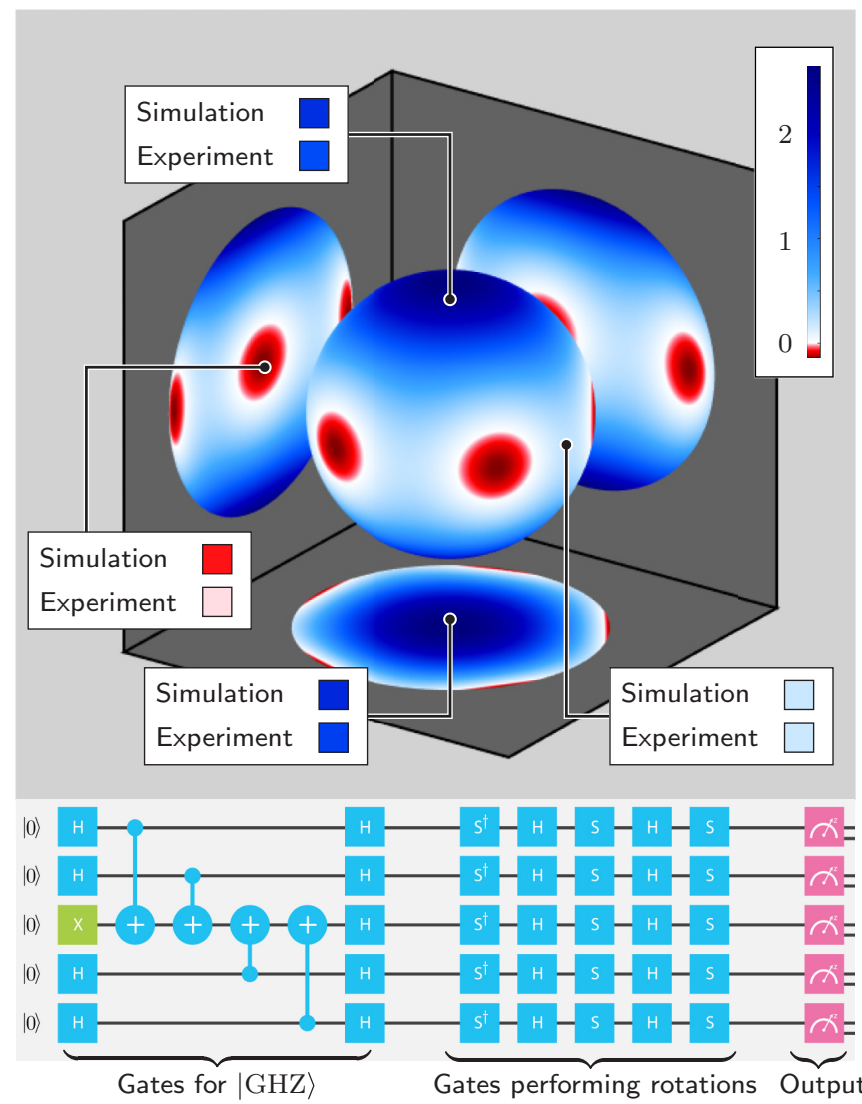

FIG. 4. Here we show the five-qubit GHZ spin Schrödinger cat state Wigner function $W_{\mathrm{SU}\left(2^{5}\right)}$ for the $\theta_{1}=\theta_{2}=\theta_{3}=\theta_{4}=\theta_{5}$ and $\varphi_{1}=\varphi_{2}=\varphi_{3}=\varphi_{4}=\varphi_{5}$ slice. This can be considered a qubitsystem analog of Fig. 1 and which was presented in Ref. [5] to reconstruct nonclassical cavity field states. We note that in Ref. [5] the interference terms that were observed correspond to quantum coherence in macroscopically distinct superpositions of states. In this figure, the interference terms should be interpreted as a direct visualization of the entanglement in the system. Here we show the ideal function, and as insets, show both simulated and experimental results from IBM's Quantum Experience project. In this figure we also show an example circuit used to generate simulated and experimental data. As with the circuits used to create the Bell states presented in Fig. 3, these gate operations ideally would be replaced by optimized, single-rotation, operations that have very recently been made available by IBM. We note that the two, nonpolar, points can be obtained in a variety of ways. Specifically they could be found by using just $\theta$ rotations, or through a combination of $\theta$ and $\varphi$ rotations. We have verified that the results that we obtained from the IBM Quantum Experience project are independent of the combination of rotations used.

motivated on an individual qubit level; a global $\pi$ rotation on each qubit's Bloch sphere. This leads to an extended parity operator that is nothing more than the tensor product of the parities of individual qubits:

$$
\Pi_{\otimes^{N} \mathrm{SU}(2)}=\bigotimes_{i=1}^{N} \Pi_{\mathrm{SU}\left(2^{[1]}\right)}^{(i)}=\bigotimes_{i=1}^{N} \frac{1}{2}\left(\mathbb{1}+\sqrt{3} \sigma_{z_{i}}\right)
$$

which for one qubit is equal to Eq. (6) but for two qubits takes the explicit form

$$
\begin{aligned}
\Pi_{\otimes{ }^{2} \mathrm{SU}(2)} & =\frac{1}{2}\left(\begin{array}{cccc}
2+\sqrt{3} & 0 & 0 & 0 \\
0 & -1 & 0 & 0 \\
0 & 0 & -1 & 0 \\
0 & 0 & 0 & 2-\sqrt{3}
\end{array}\right) \\
& =\frac{1}{4}\left[\mathbb{1} \otimes \mathbb{1}+\sqrt{3} \mathbb{1} \otimes \sigma_{z}+\sqrt{3} \sigma_{z} \otimes \mathbb{1}+3 \sigma_{z} \otimes \sigma_{z}\right]
\end{aligned}
$$

in the computational basis. When compared with Eq. (7) we see that this version of extended parity no longer treats one-qubit and two-qubit contributions on an equal footing. The definition of the Wigner function continues in the same way as before and, in terms of the rotated density matrix $\tilde{\rho}=\mathbb{U}_{N}^{\dagger} \rho \mathbb{U}_{N}$, takes the form

$$
\begin{aligned}
W_{\bigotimes^{N} \mathrm{SU}(2)}(\boldsymbol{\Omega}) & =\operatorname{Tr}\left[\tilde{\rho}(\boldsymbol{\Omega}) \Pi_{\bigotimes^{N} \mathrm{SU}(2)}\right] \\
& =\sum_{n} \tilde{\rho}_{n n}(\boldsymbol{\Omega})\left(\Pi_{\bigotimes^{N} \mathrm{SU}(2)}\right)_{n n} .
\end{aligned}
$$

Returning to Figs. 2(e) and 2(f) we show example slices of $W_{\otimes^{N} \operatorname{SU}(2)}^{\theta_{i}=0}\left(\theta_{1}, \theta_{2}\right)=W_{\otimes^{N} \operatorname{SU}(2)}\left(\theta_{1}, \varphi_{1}=0, \theta_{2}, \varphi_{2}=0\right)$ that demonstrate that this alternative Wigner function is qualitatively very similar to the equivalent slices of $W_{\mathrm{SU}\left(2^{[N]}\right)}(\Omega)$ shown in Figs. 2(c) and 2(d) [46].

In Fig. 5 (top) we show results for comparison with Fig. 4 and (bottom) (and, by analogy, with nonclassical cavity field states [5]) with Fig. 3 which demonstrates that $W_{\otimes^{N} S U(2)}$ is a Wigner function with qualitatively very similar features to $W_{\mathrm{SU}\left(2^{[N]}\right)}$ that will be compared in the next section. For the top and bottom point the theoretical value is 2.375 . The simulated values are 1.13 and 1.11, and the experimental values are 0.8876 and 0.9006 , respectively.

\section{EFFICIENT STATE ESTIMATION, CHARACTERISATION, AND ENTANGLEMENT VALIDATION}

As they are informationally complete, our Wigner functions for spin can be considered mathematically equivalent to the density matrix (state space) formulation. They also exhibit unique and intuitively natural characteristic features. If, for example, we look at Fig. 5 for the GHZ state (which is a superposition of spin coherent states) it is clear that there are regions of strong oscillations in the equal-angle slice; these are reminiscent of the interference terms between two harmonic-oscillator coherent states shown in Fig. 1. It is natural to ask if measurement of such characteristic features can be used to verify nonclassical properties of the state such as quantum coherence or entanglement. In other words, can we extract information in a similar way as for Wigner functions of continuous systems where negativity is a signature of nonclassical correlations? In finite-dimensional systems things are a little more complicated because negativity of the Wigner function has some subtle complexities which we will expand on later in this paper and in full detail in a later work. Moreover, the exact form of a state's spin Wigner function is 


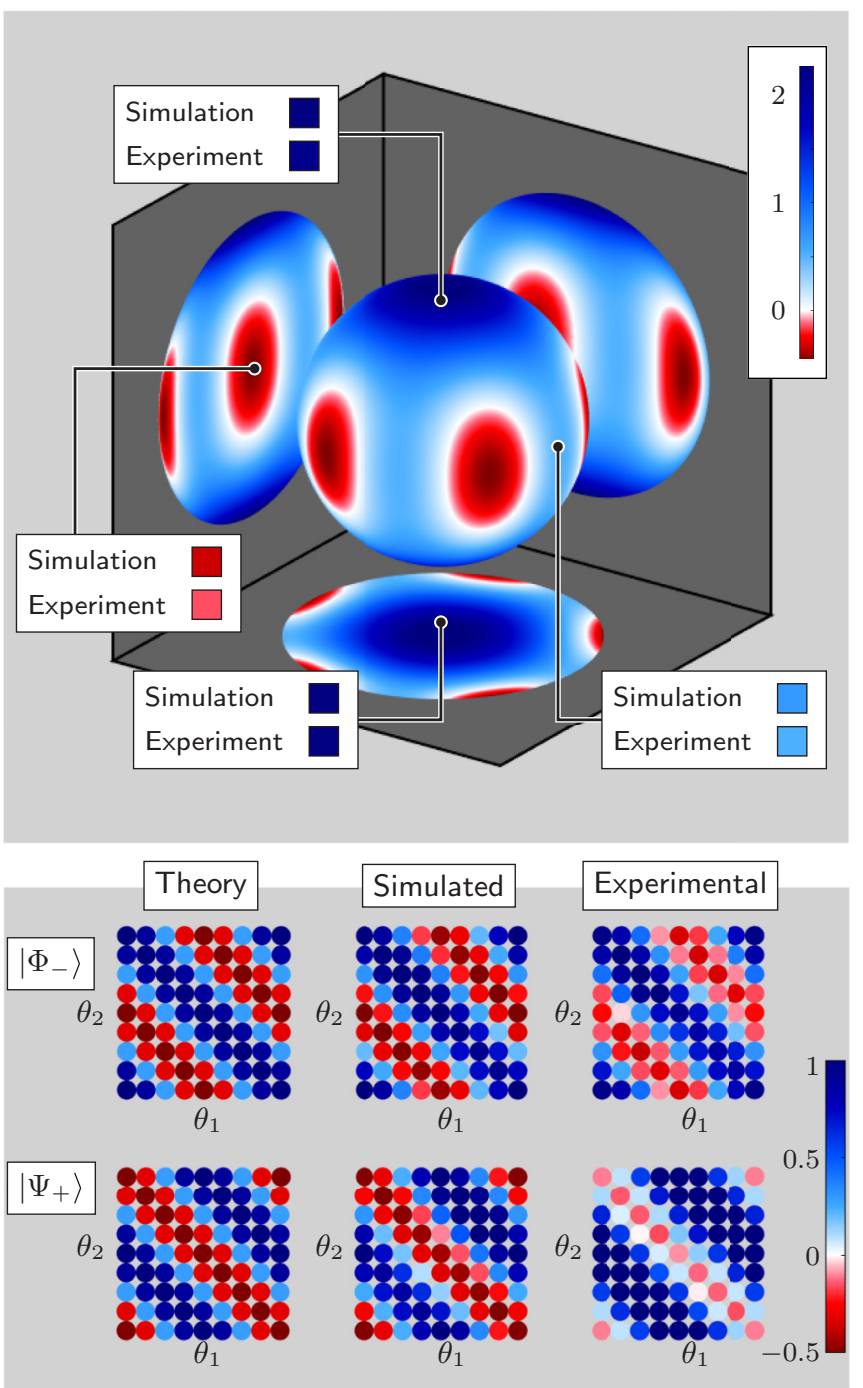

FIG. 5. Here we reproduce Figs. 3 and 4 by using the same data but now employing the Wigner function defined by using the alternative extended parity operators as given in Eq. (12). In the top figure, for comparison with Fig. 4, we show the five-qubit GHZ spin Schrödinger cat state Wigner function $W_{\otimes^{5} \mathrm{SU}(2)}$ for the $\theta_{1}=\theta_{2}=\theta_{3}=\theta_{4}=\theta_{5}$ and $\varphi_{1}=\varphi_{2}=\varphi_{3}=\varphi_{4}=\varphi_{5}$ slice. Again we show the ideal function and, as insets, show both simulated and experimental results from IBM's Quantum Experience project. On the bottom figure, for comparison with Fig. 3, we provide plots of $W_{\otimes^{2} \mathrm{SU}(2)}$ for the two Bell states $\left|\Phi_{+}\right\rangle$and $\left|\Psi_{-}\right\rangle$. We plot $\theta_{1}$ versus $\theta_{2}$ for the $\varphi_{1}=\varphi_{2}=0$ slice of the Wigner function for two qubits. Once more, we have included for comparison ideal theoretical values, numerical results using IBM's built in simulator, and real experimental data from IBM's quantum processor. Again we see good agreement between theory, simulation and experiment and note that using a different extended parity operator provides an alternative path to direct measurement of phase space.

fixed by the chosen extended parity operator that is used. As such, it may be that different extended parity operations may be more or less useful in revealing particular characteristic features of the quantum state. To focus the discussion in this section we fix our choice of parity and Wigner function to $\Pi_{\otimes^{N} \mathrm{SU}(2)}$ and $W_{\bigotimes^{N} \mathrm{SU}(2)}$. We discuss with reference to this specific Wigner function possibilities for efficient state characterization or categorization (e.g., by identifying features peculiar to GHZ states). We show that if one has sufficient prior information about the expected state of the system (such as that it comprises a superposition of antipodal spin coherent states) it may be possible to validate entanglement with only a couple of measurements.

To begin we consider the $N$-qubit state

$$
\rho(\gamma)=\gamma \rho_{\mathrm{GHZ}}+(1-\gamma) \rho_{m},
$$

where $\gamma \in[0,1]$. Here $\rho(\gamma)$ interpolates between the density operators $\rho_{\mathrm{GHZ}}$ for the GHZ state (the coherent superposition of $|11111\rangle$ and $|00000\rangle$ with $\gamma=1)$ and $\rho_{m}$ for the statistical mixture of $|11111\rangle$ and $|00000\rangle$ (with $\gamma=0$ ). The Wigner function of this state is

$$
\begin{aligned}
W_{\otimes^{N} \mathrm{SU}(2)}^{(\gamma)}(\boldsymbol{\Omega})= & \frac{1}{2^{N+1}} \prod_{i=1}^{N}\left(1+\sqrt{3} \cos 2 \theta_{i}\right) \\
& +\frac{1}{2^{N+1}} \prod_{i=1}^{N}\left(1-\sqrt{3} \cos 2 \theta_{i}\right) \\
& -\frac{\gamma}{2^{N}} \prod_{i=1}^{N}\left(-\sqrt{3} \sin 2 \theta_{i}\right) \cos \left(2 \sum_{i}^{N} \varphi_{i}\right) .
\end{aligned}
$$

When $\gamma=1$ we can see that the $N$-qubit GHZ state is made up of three terms: the first two correspond to the first and last diagonal elements of the density matrix in Eq. (13) and the third (interference) term to the maximally off-diagonal elements. Figure 6 compares the equal-angle Wigner functions $(\theta=$ $\left.\theta_{1}=\cdots=\theta_{N}, \varphi=\varphi_{1}=\cdots=\varphi_{N}\right)$ of the GHZ state $\gamma=1$ [Fig. 6(a)] and the separable mixed state $\gamma=0$ [Fig. 6(b)]. As can be seen, the maxima at the top and bottom of the sphere are the same in both states, although the equatorial oscillations are absent in the separable state.

From this simple example, it is clear that the oscillations around the equator, where all $\theta_{i}=\pi / 4$, arise entirely from the $\cos \left(2 \sum_{i}^{N} \varphi_{i}\right)$ term. These oscillations, which are of maximum possible frequency for a Wigner function with this number of qubits, are characteristic of GHZ-type superposition (compare the iconic Wigner function Fig. 1) and are analogous to the super-resolution oscillations observed in NOON states [53]. We note that any antipodal superposition of spin coherent states will be look like a rotated version of Fig. 6(a) with interference terms along the geodesic bisecting them. It is natural to ask if such oscillations can be used to certify GHZ-type entanglement. We note that negativity in the Wigner function alone is insufficient to be a signal of entanglement. To illustrate this we show in Fig. 6(c) the equal-angle slice Wigner function for the state $|10000\rangle$ and note that, despite being separable, it has significant negativity in this equal-angle slice. Indeed, the equal-angle slice of the $W_{\otimes^{5} \mathrm{SU}(2)}$ function for the statistical mixture of $|10000\rangle,|01000\rangle$, etc. is identical to Fig. 6(c). To establish if there is a potential to use the characteristic features of the GHZ Wigner function equal-angle slice for certification we can ask what is the nearest separable state in terms of its phase-space characteristics. We believe the closest in form is 


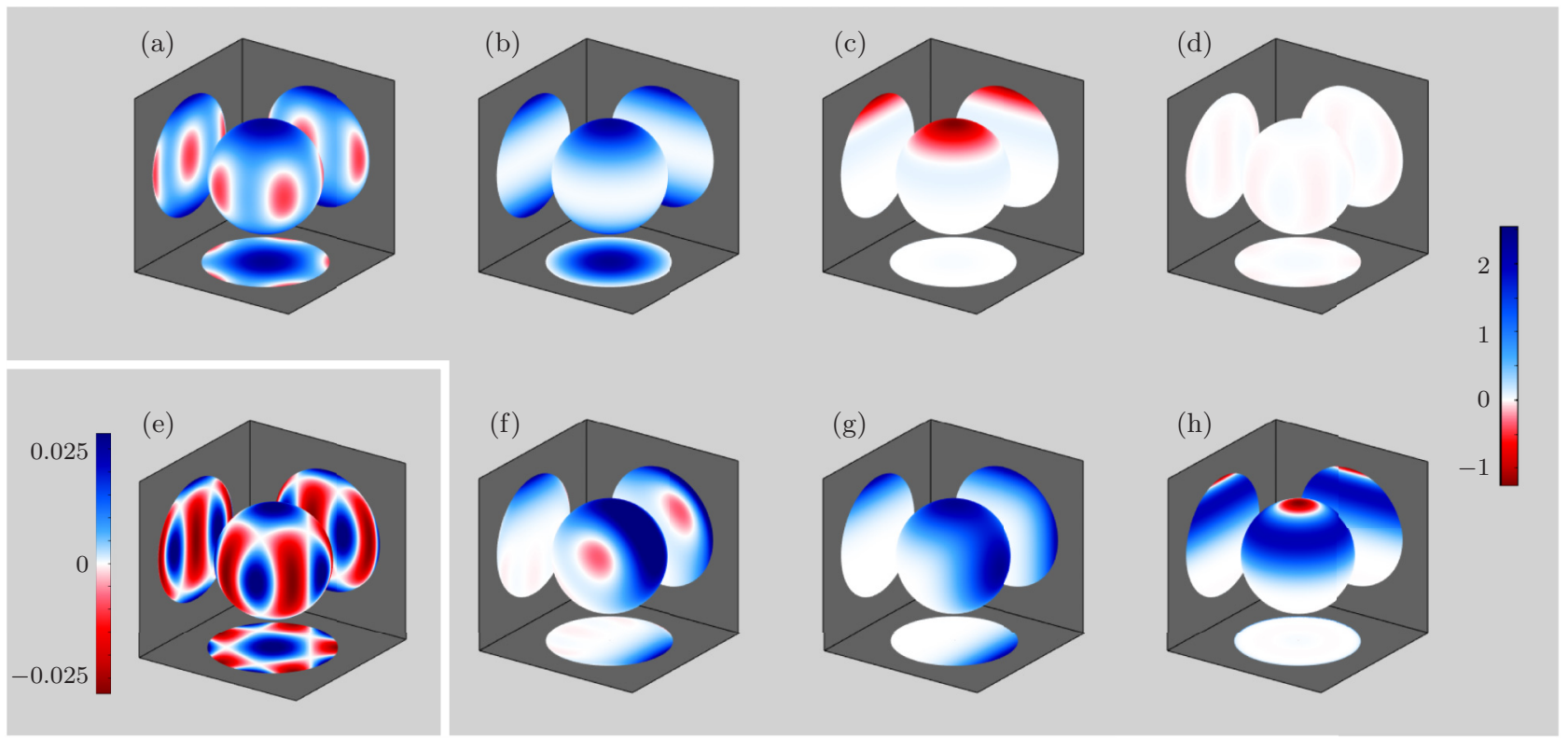

FIG. 6. Here we show the equal-angle slice $W_{\otimes^{N} \mathrm{SU}(2)}$ Wigner function for various 5-qubit states. Panel (a) shows the same GHZ state as seen in Fig. 5, with panel (b) showing the mixed state counterpart of this GHZ state given by $\left[(|0\rangle\langle 0|)^{\otimes N}+(|1\rangle\langle 1|)^{\otimes N}\right] / 2$ Only the pure state displays the interference pattern given by the off-diagonal terms in the density matrix when the state is entangled. In panel (c) we see the state $|10000\rangle$. In panels (d) and (e) we see the clock state; panel (d) is shown with the same color map as the other plots, whereas panel (e) shows the state with a modified color map to show the structure of the slice that is not evident with the color maps used throughout the rest of this figure. (f), (g) Defining $|\rightarrow\rangle=(|0\rangle+|1\rangle) / \sqrt{2}$ we show the entangled superposition of spin coherent states $|0\rangle^{\otimes N}+|\rightarrow\rangle^{\otimes N}$ and its mixed-state counterpart, the equally weighted statistical mixture of $(|0\rangle\langle 0|)^{\otimes N}$ and $(|\rightarrow\rangle\langle\rightarrow|)^{\otimes N}$. Again note that only the pure state has negative interference terms in this slice with two of particularly large amplitude. Finally, panel (h) shows the equal-angle slice Wigner function for the five-qubit $W$ state showing that other entangled states have patterns that could also act as state identification signatures.

the "clock" state which we define by

$$
\left|\psi_{\text {clock }}\right\rangle=\frac{1}{2^{N / 2}} \bigotimes_{k=1}^{N}\left[|0\rangle+\exp \left(\frac{2 \mathrm{i} \pi k}{N}\right)|1\rangle\right],
$$

whose Wigner function is

$$
W_{\otimes^{N} \mathrm{SU}(2)}^{\text {clock }}(\boldsymbol{\Omega})=\frac{1}{2^{N}} \prod_{k=1}^{N} 1+\sqrt{3} \sin 2 \theta_{k} \cos \left(2 \varphi_{k}+\frac{2 \pi k}{N}\right) .
$$

We show the equal-angle slice of this function in Fig. 6(d). We note that there is a similar oscillatory character to that seen in the GHZ state but that it is exponentially smaller in amplitude. For this reason we show this function again in Fig. 6(e) but on a different scale. It is straightforward to show ${ }^{4}$ therefore that oscillations of this wavelength that exceed those of the clock state Wigner oscillations is a signature of a GHZ type of entanglement-something that in principle can be established with only two measurements. Due to an update on the IBM computer during the closing stages of our work, we were able

\footnotetext{
${ }^{4}$ The maximum-frequency equatorial oscillations of the Wigner function are determined by the top-right and bottom-left elements of the density matrix. The maximum amplitude of these for any product state $\bigotimes_{k=1}^{N}\left(a_{k}|0\rangle+b_{k}|1\rangle\right)$ occurs when $\left|a_{k}\right|=\left|b_{k}\right|=1 / \sqrt{2}$ and has magnitude $2^{-N}$, compared with $2^{-1}$ for the GHZ state.
}

to observe these oscillations directly, as seen in Fig. 7. This was due to the implementation of three new gates that can perform arbitrary rotations on individual qubits: $u_{1}(\lambda), u_{2}(\tilde{\varphi}, \lambda)$, and $u_{3}(\tilde{\theta}, \tilde{\varphi}, \lambda)$, with $u_{3}(\tilde{\theta}, \tilde{\varphi}, \lambda)=e^{-\mathrm{i} \tilde{\varphi} \sigma_{z} / 2} e^{-\mathrm{i} \tilde{\theta} \sigma_{y} / 2} e^{-\mathrm{i} \lambda \sigma_{z} / 2}$. These three gates give us the freedom to specify any rotation or any point in phase space in $S U(2)$, allowing us to sweep around the equator, experimentally verifying the presence of the interference-based oscillation for a 5-qubit GHZ state. In Fig. 7 these measurement results are compared with ideal theoretical values. The experimentally measured amplitudes are somewhat reduced, as well as having an offset phase. This indicates that the computer is not producing a perfect GHZ state but that the state is verified to be both entangled and in reasonably consistent agreement with a perfect GHZ state. However, since there is a linear mapping between the density matrix and the Wigner function, a similar Wigner function implies the state is at least similar, making feature-based characterization robust against small errors in state preparation and limited decoherence, likely candidates for the difference between the theoretical and experimental curves.

The advantage of our approach is in the potential to reduce the number of measurements required to develop confidence certifying more general states. For example, begin by defining $|\rightarrow\rangle=(|0\rangle+|1\rangle) / \sqrt{2}$. We then generate the normalized equal superposition of $|0\rangle^{\otimes N}$ and $|\rightarrow\rangle^{\otimes N}$. In Fig. 6(f) we show $W_{\otimes^{N} S U(2)}$ for this state and the corresponding equally weighted mixture of $|0\rangle^{\otimes N}$ and $|\rightarrow\rangle^{\otimes N}$ [Fig. 6(g)]. Both density matrices have no nonzero elements 


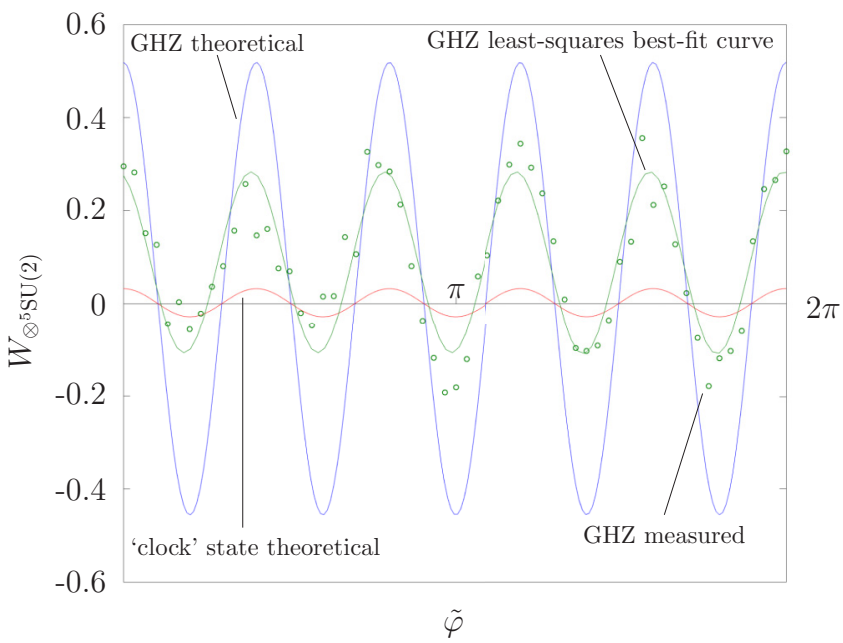

FIG. 7. Points around the equator of the 5-qubit GHZ state Wigner function from Fig. 5 with $\theta_{1}=\theta_{2}=\cdots=\theta_{N}=\frac{\pi}{4}, \varphi_{1}=$ $\varphi_{2}=\cdots=\varphi_{N}=\varphi$ where the blue curve is the theoretically calculated values for an ideal GHZ state found from our model. The green dots are the measured experimental results and the green curve is a least-square best-fit sinusoid to the experimental results. In red we see the oscillations given around the equator for the separable clock state of Eq. (15); the amplitude is significantly lower than for the "GHZ measured" state demonstrating that the latter (whose oscillations are not as strong as the theoretical maximum) is indeed entangled. Here, $\tilde{\varphi}=2 \varphi$ to correspond to how IBM defines the rotations on their machine.

in the computational basis (making conventional tomography challenging), but the superposition can be certified to be within an acceptable confidence interval through a few measurements of the characteristic features of its corresponding Wigner function. As with our analysis of the GHZ state the presence of this structure may be used to give confidence in the existence of entanglement in the system and categorization as a superposition of spin coherent states. As another example, we show in Fig. 6(h) the equal-angle Wigner function for the $W$ state of five qubits. Again we see that $W$ states have a distinctive shape (because $W$ states can be thought of as "eigenstates of a total ' $z$ ' angular-momentum operator," this form is intuitively sensible). Once more, it would not take more than a few measurements to gain significant confidence that a system was or was not in a $W$ state.

In terms of the more general problem of rapid quantum state estimation, spin-Wigner functions may be of particular use when some properties of the state are known in advance. We have already noted that only a few measurements are needed to verify that certain characteristic features of the Wigner function are present. Because it is possible to build these Wigner functions from expansions by using bases other than the computational basis, such as from stabilizer states, full quantum-state reconstruction can be viewed as establishing the coefficients of such expansions. Understanding the structure of these expansions together with foreknowledge of the set of potential states a system may achieve can lead to efficient state-estimation protocols. This is because one can select measurements that rapidly exclude very many of the components of the expansion and confirm the presence of the dominant terms. In this way phase-space methods provide an alternative path to efficient state estimation from those known in other areas of quantum state tomography [54-58]. A detailed study of efficient quantum state reconstruction in phase space will be the subject of a future work.

\section{ON THE DIFFERENCES BETWEEN WIGNER FUNCTIONS}

Each of the two cases we have considered here have their own strengths which will be expanded on in a later publication. However, we are including a brief discussion to highlight that there is some freedom in choosing extended parity operators in tensor product spaces. This should be of utility because it increases the number of available options in designing experiments for the direct measurement of a Wigner function.

The full-group Wigner function $W_{\mathrm{SU}\left(2^{[N]}\right)}$ and the tensorproduct Wigner function $W_{\bigotimes^{N} S U(2)}$ are related to the density matrix by different, but still invertible, linear maps, and therefore both contain full information about the quantum state. The tensor-product form has the additional property of respecting the marginals in each subspace. We can see that this is indeed the case by noting that the two-qubit kernel separates,

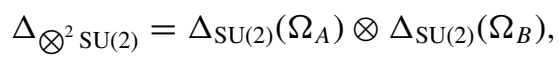

leading to the result

$$
\begin{aligned}
\int W_{\otimes^{2} \mathrm{SU}(2)}\left(\Omega_{A}, \Omega_{B}\right) \mathrm{d} \Omega_{B} & =\operatorname{Tr}\left[\rho_{A} \Delta_{\mathrm{SU}(2)}\left(\Omega_{A}\right)\right] \\
& =W_{\mathrm{SU}(2)}\left(\Omega_{A}\right),
\end{aligned}
$$

where $\rho_{A}$ is the reduced density matrix of subsystem $A$. Note that extension to an arbitrary number of qubits is a trivial extension of this argument.

As an example, consider the Bell state $\left|\Psi_{+}\right\rangle$shown in Fig. 2(b). Here our two Wigner-function cases have the same structure, with the tensor-product form having a larger amplitude of modulation:

$$
\begin{aligned}
& W_{\mathrm{SU}\left(2^{[2]}\right)}=\frac{1}{4}\left[1+\sqrt{5}\left(x_{A} x_{B}+y_{A} y_{B}-z_{A} z_{B}\right)\right], \\
& W_{\otimes^{2} \mathrm{SU}(2)}=\frac{1}{4}\left[1+3\left(x_{A} x_{B}+y_{A} y_{B}-z_{A} z_{B}\right)\right],
\end{aligned}
$$

where $\left(x_{i}, y_{i}, z_{i}\right)$ is the unit vector in the direction $\Omega_{i}$. However, for the product state $\left(|0\rangle_{1}|0\rangle_{2}\right)$ we see a distinction in angular dependence:

$$
\begin{aligned}
& W_{\mathrm{SU}\left(2^{[2]}\right)}=\frac{1}{4}\left[1+\sqrt{5}\left(z_{A}+z_{B}\right)+\sqrt{5} z_{A} z_{B}\right], \\
& W_{\otimes^{2} \mathrm{SU}(2)}=\frac{1}{4}\left[1+\sqrt{3}\left(z_{A}+z_{B}\right)+3 z_{A} z_{B}\right] .
\end{aligned}
$$

Note that the one-qubit and two-qubit angular terms carry coefficients of different magnitude in the tensor-product Wigner function.

The above distinctions have led us to speculate that the two different forms of the Wigner function that we consider in this paper may be useful as a mechanism to differentiate (in representation) logical and physical qubit systems. That is, when there is a natural separation into physical qubits, into subsystems, or into a system and an environment, we choose the tensor product formulation. If, on the other hand, the system under consideration comprises a many-level quantum system 
constrained to act as logical qubits, it is less natural to impose a tensor product structure to the phase-space representation than use the full-group form, which may be more natural. Furthermore, in systems that comprise a mixture of logical and physical qubits a tensor product of the different kernels could be used to maintain this distinction. We also note that in the case of $W_{\otimes^{2} \mathrm{SU}(2)}$ the Weyl transform $\rho=\int_{\boldsymbol{\Omega}} \mathrm{W}_{\rho}(\boldsymbol{\Omega}) \Delta(\boldsymbol{\Omega}) \mathrm{d} \boldsymbol{\Omega}$ reconstructs the original density matrix but in the case of $W_{\mathrm{SU}\left(2^{[2]}\right)}$ a further linear transform is needed. If reconstruction of the density matrix from the Wigner function is desired, $W_{\otimes^{2} \mathrm{SU}(2)}$ would be the more appropriate choice. While much further work needs to be done, it may well be that drawing such distinctions may help us understand separability from a phase-space perspective, thus enabling derivation of new useful entanglement measures.

\section{CONCLUDING REMARKS}

We have demonstrated a simple method for quantum state reconstruction that extends those previously known for quantum optical systems [5-10] to other classes of systems. Using IBM's Quantum Experience five-qubit quantum processor, we have shown reconstruction of two Bell states and the five-qubit GHZ spin Schrödinger cat state via spin Wigner function measurements. We note that our procedure could be made much more efficient by direct implementation of rotation operations and measurement of any suitable extended parity operator (or, if appropriate, direct measurement of the rotated extended parity). By doing so, the potential advantage of our procedure over other tomographic methods would be made much clearer in that fewer measurements would be needed to check certain properties of the quantum state. In such an instance, in verifying the fidelity of a high-quality GHZ state, only a small set of measurements that quantifies the strength of the interference terms is needed, providing an improvement over traditional quantum state tomography. Furthermore, this work demonstrates how phase-space methods can be of utility in understanding processes such as decoherence and be useful in the "debugging" of quantum information processors. In particular we have proposed a method for verifying whether a system is entangled that uses only a few measurements and which in some circumstances, where suitable constraints of the range of possible states are known, may potentially be reduced to only two. The utility of this work extends beyond metrology because the inclusion of tomography in device engineering will no doubt be of use in the development of quantum analogs for "Design for Test," debug, fault identification, and system certification.

\section{ACKNOWLEDGMENTS}

We would like to thank Jay Gambetta, Lev Bishop, Ray Bishop, and Simon Devitt for interesting and informative discussions. We are deeply grateful to IBM's Quantum Computing research team and the IBM Quantum Experience project which made it possible for us to easily obtain experimental results. R.P.R. received support from EPSRC Grant No. EP/N509516/1.
[1] E. Wigner, Phys. Rev. 40, 749 (1932).

[2] E. C. G. Sudarshan, Phys. Rev. Lett. 10, 277 (1963).

[3] R. J. Glauber, Phys. Rev. 131, 2766 (1963).

[4] H. J. Carmichael, Statistical Methods in Quantum Optics I (Springer-Verlag, Berlin, 2002).

[5] S. Deléglise, I. Dotsenko, C. Sayrin, J. Bernu, M. Brune, J. M. Raimond, and S. Haroche, Nature (London) 455, 510 (2008).

[6] L. G. Lutterbach and L. Davidovich, Phys. Rev. Lett. 78, 2547 (1997).

[7] K. Banaszek, C. Radzewicz, K. Wódkiewicz, and J. S. Krasiński, Phys. Rev. A 60, 674 (1999).

[8] P. Bertet, A. Auffeves, P. Maioli, S. Osnaghi, T. Meunier, M. Brune, J. M. Raimond, and S. Haroche, Phys. Rev. Lett. 89, 200402 (2002).

[9] A. I. Lvovsky, H. Hansen, T. Aichele, O. Benson, J. Mlynek, and S. Schiller, Phys. Rev. Lett. 87, 050402 (2001).

[10] A. Zavatta, S. Viciani, and M. Bellini, Phys. Rev. A 70, 053821 (2004).

[11] C. C. Gerry and J. Mimih, Contemp. Phys. 51, 497 (2010).

[12] C. C. Gerry, A. Benmoussa, and R. A. Campos, Phys. Rev. A 72, 053818 (2005).

[13] J. P. Dowling, G. S. Agarwal, and W. P. Schleich, Phys. Rev. A 49, 4101 (1994).

[14] F. T. Arecchi, E. Courtens, R. Gilmore, and H. Thomas, Phys. Rev. A 6, 2211 (1972).

[15] Y. Kano, J. Phys. Soc. Jpn. 36, 39 (1974).

[16] K. E. Cahill and R. J. Glauber, Phys. Rev. 177, 1882 (1969).
[17] G. S. Agarwal, Phys. Rev. A 24, 2889 (1981).

[18] J. E. Moyal, Math. Proc. Cambridge Philos. Soc. 45, 99 (1949).

[19] R. H. Dicke, Phys. Rev. 93, 99 (1954).

[20] R. Friedberg and J. T. Manassah, Laser Phys. Lett. 4, 900 (2007).

[21] K. Nemoto, J. Phys. A: Math. Gen. 33, 3493 (2000).

[22] M. Mathur and H. S. Mani, J. Math. Phys. 43, 5351 (2002).

[23] N. M. Atakishiyev, S. M. Chumakov, and K. B. Wolf, J. Math. Phys. 39, 6247 (1998).

[24] A. Luis, Phys. Rev. A 69, 052112 (2004).

[25] A. Luis, J. Phys. A: Math. Theor. 41, 495302 (2008).

[26] A. B. Klimov and J. L. Romero, J. Phys. A: Math. Theor. 41, 055303 (2008).

[27] T. Tilma and K. Nemoto, J. Phys. A: Math. Theor. 45, 015302 (2011)

[28] R. L. Stratonovich, Sov. Phys. JETP 31, 1012 (1956).

[29] Y. Takahashi and F. Shibata, J. Stat. Phys. 14, 49 (1976).

[30] C. Brif and A. Mann, Phys. Rev. A 59, 971 (1999).

[31] M. K. Patra and S. L. Braunstein, New J. Phys. 13, 063013 (2011).

[32] A. B. Klimov and H. de Guise, J. Phys. A: Math. Theor. 43, 402001 (2010).

[33] W. K. Wootters, Ann. Phys. (NY) 176, 1 (1987).

[34] L. J. Boya, E. C. G. Sudarshan, and T. Tilma, Rep. Math. Phys. 52, 401 (2003).

[35] T. Tilma and E. C. G. Sudarshan, J. Geom. Phys. 52, 263 (2004).

[36] T. Tilma, M. J. Everitt, J. H. Samson, W. J. Munro, and K. Nemoto, Phys. Rev. Lett. 117, 180401 (2016). 
[37] J. R. Klauder and E. C. G. Sudarshan, Fundamentals of Quantum Optics (W. A. Benjamin, New York, 1968); reprinted by Dover Publishing (2006).

[38] M. Hillery, R. F. O'Connell, M. O. Scully, and E. P. Wigner, Phys. Rep. 106, 121 (1984).

[39] H. Weyl, Eur. Phys. J. A 46, 1 (1927); Gruppenrheorie and Quantcnmechanik (S. Hirzel Verlag, Leipzig, 1931); English reprint (Dover Publications, New York, 1950), p. 275.

[40] J. C. Várilly and J. Gracia-Bondía, Ann. Phys. (NY) 190, 107 (1989).

[41] U. Leonhardt, Phys. Rev. A 53, 2998 (1996).

[42] A. Vourdas, Rep. Math. Phys. 40, 367 (1997).

[43] C. Miquel, J. P. Paz, and M. Saraceno, Phys. Rev. A 65, 062309 (2002).

[44] K. S. Gibbons, M. J. Hoffman, and W. K. Wootters, Phys. Rev. A 70, 062101 (2004).

[45] R. F. Bishop and A. Vourdas, Phys. Rev. A 50, 4488 (1994).

[46] See Supplemental Material at http://link.aps.org/supplemental/ 10.1103/PhysRevA.96.022117 for animation_01 where we show the $W_{\mathrm{SU}\left(2^{[2]}\right)}$-Wigner function dynamics for the Deutsch algorithm for two qubits where the $U_{f}$ gate is a CNOT gate, for animation_02, where we show the $W_{\mathrm{SU}\left(2^{[2]}\right)}$-Wigner function dynamics for the creation of the four Bell states, and for animation_03, where we show a $W_{\otimes^{2} \text { SU(2) }}$-Wigner function version of animation_02 for comparative purposes.
[47] D. Deutsch and R. Jozsa, Proc. R. Soc. London, Ser. A 439, 553 (1992).

[48] S. J. Devitt, Phys. Rev. A 94, 032329 (2016).

[49] D. Alsina and J. I. Latorre, Phys. Rev. A 94, 012314 (2016).

[50] D. M. Greenberger, M. A. Horne, and A. Zeilinger, in Bell's Theorem, Quantum Theory, and Conceptions of the Universe, edited by M. Kafatos (Kluwer, Dordrecht, 1989), pp. 69-72.

[51] M. J. Everitt, W. J. Munro, and T. P. Spiller, Phys. Rev. A 85, 022113 (2012).

[52] S. Dooley, F. McCrossan, D. Harland, M. J. Everitt, and T. P. Spiller, Phys. Rev. A 87, 052323 (2013).

[53] A. N. Boto, P. Kok, D. S. Abrams, S. L. Braunstein, C. P. Williams, and J. P. Dowling, Phys. Rev. Lett. 85, 2733 (2000).

[54] M. Cramer, M. B. Plenio, S. T. Flammia, R. Somma, D. Gross, S. D. Bartlett, O. Landon-Cardinal, D. Poulin, and Y.-K. Liu, Nat. Commun. 1, 149 (2010).

[55] B. P. Lanyon, C. Maier, M. Holzpfel, T. Baumgratz, C. Hempel, P. Jurcevic, I. Dhand, A. S. Buyskikh, A. J. Daley, M. Cramer, M. B. Plenio, R. Blatt, and C. F. Roos, arXiv:1612.08000.

[56] T. Baumgratz, A. Neler, M. Cramer, and M. B. Plenio, New J. Phys. 15, 125004 (2013).

[57] T. Baumgratz, D. Gross, M. Cramer, and M. B. Plenio, Phys. Rev. Lett. 111, 020401 (2013).

[58] M. Cramer, M. B. Plenio, and H. Wunderlich, Phys. Rev. Lett. 106, 020401 (2011). 